Revue de droit comparé du travail et de la sécurité sociale

3 | 2017

Le travail dans l'économie informelle, un défi pour le droit social

\title{
La part du droit dans la formalisation de l'emploi en Algérie : une diversité de mesures, sans stratégie claire
}

Mahammed Nasr-eddine Koriche

\section{OpenEdition}

Journals

Édition électronique

URL : https://journals.openedition.org/rdctss/358

DOI : $10.4000 /$ rdctss.358

ISSN : 2262-9815

Éditeur

Centre de droit comparé du travail et de la sécurité sociale

Édition imprimée

Date de publication : 1 septembre 2017

Pagination : 78-89

ISSN : 2117-4350

Référence électronique

Mahammed Nasr-eddine Koriche, «La part du droit dans la formalisation de l'emploi en Algérie : une diversité de mesures, sans stratégie claire ", Revue de droit comparé du travail et de la sécurité sociale [En ligne], 3 | 2017, mis en ligne le 01 novembre 2018, consulté le 04 décembre 2021. URL : http:// journals.openedition.org/rdctss/358; DOI : https://doi.org/10.4000/rdctss.358

\section{(c) $(1)$}

Revue de droit comparé du travail et de la sécurité sociale est mise à disposition selon les termes de la Licence Creative Commons Attribution - Pas d'Utilisation Commerciale - Pas de Modification 4.0 International. 


\section{LA PART DU DROIT DANS LA FORMALISATION DE L'EMPLOI EN ALGÉRIE :}

UNE DIVERSITÉ DE MESURES, SANS STRATÉGIE CLAIRE

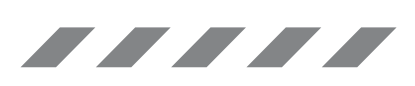

\section{ABSTRACT}

To reduce informal work, State intervention is necessary to organise the labour market and encourage the creation and declaration of jobs. Above all, this formalisation entails increasing the government's ability to enforce existing laws and ensure that labour law can be extended to particular work situations.

KEYWORDS: Informal work, wage labour, independent work, extension of labour law.

\section{RÉSUMÉ}

Pour réduire l'informalité du travail, l'intervention de l'Etat est nécessaire afin d'organiser le marché du travail et d'encourager la création et la déclaration des emplois. Cependant, la formalisation signifie surtout accroître les capacités à faire appliquer les lois existantes et à assurer l'extension du champ d'application du droit social à des situations particulières de travail.

MOTS CLÉS: Travail informel, travail salarié, travail indépendant, extension du droit social. 


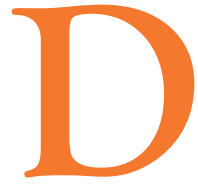

ans la Recommandation $\mathrm{n}^{\circ} 204$ de l'OIT sur la transition de l'économie informelle vers l'économie formelle (2015), l'expression « économie informelle » se rapporte «à toutes les activités économiques des travailleurs et des unités économiques qui - en droit ou en pratique - ne sont pas couvertes ou sont insuffisamment couvertes par des dispositions formelles ${ }^{01} \gg$.

En Algérie, toutes les activités marchandes, quelle que soit la taille des unités économiques, ont vocation à entrer dans le champ d'application de dispositions formelles légales et réglementaires (code de commerce, registre de commerce, code des impôts, législation du travail et de la sécurité sociale, etc.). Si parmi elles, il en est qui opèrent en dehors de la loi, c'est que les services de l'Administration publique, en charge de l'application des lois dans leurs secteurs respectifs, sont défaillants. Ce qui signifie que c'est de facto que la loi n'est pas appliquée à ces unités économiques.

Lorsqu'il s'agit des travailleurs, le degré d'informalité est généralement apprécié en fonction de l'occupation d'un emploi ou d'une activité de qualité, de l'accès à une couverture de sécurité sociale et de la protection conférée par la législation du travail.

Le droit du travail et celui de la sécurité sociale ont d'abord été conçus pour s'appliquer aux travailleurs salariés. L'emploi salarié peut être considéré comme étant « informel » lorsque l'employeur ne procède pas aux déclarations qu'impose la législation relative aux assurances sociales et ne se conforme pas aux prescriptions de la législation du travail. Il en résulte que l'emploi salarié informel n'est pas spécifique au secteur informel. Il se retrouve également dans les entreprises du secteur formel, lorsque les travailleurs recrutés ne sont pas déclarés et que l'effectivité de la législation du travail est incertaine.

L'emploi doit également être considéré comme informel lorsque le travailleur qui exerce une activité marchande, pour son propre compte, ne se déclare pas et ne dispose donc d'aucune autorisation pour exercer légalement cette activité, en elle-même licite. La protection de ces travailleurs est problématique; elle se pose différemment selon qu'il s'agit de leur appliquer des protections tirées du droit du travail ou du droit de la sécurité sociale. En effet, la protection par le droit du travail suppose l'existence d'une relation de travail salariée là où le travailleur semblait indépendant, mais ne l'était pas réellement. La protection par le droit de la sécurité sociale est susceptible de s'étendre à toute situation de travail, que celui-ci s'exerce ou non dans le cadre d'une relation de travail salariée.

Il ne semble pas qu'une stratégie claire ait été adoptée par les pouvoirs publics pour assurer la transition vers la formalité. Toutefois, il y a bien une prise en compte, en droit algérien, de l'emploi informel et des mesures diverses sont adoptées pour réduire ce phénomène. Elles sont en rapport avec la promotion de l'emploi (I) et la protection par le droit du travail et celui de la sécurité sociale (II). D'autres politiques (dont il ne sera pas traité ici) sont préconisées pour promouvoir l'entrepreneuriat, la modernisation des entreprises, la formation professionnelle, etc.

01 Article 2 a) de la Recommandation $n^{\circ} 204$. 


\section{I - La promotion de l'emploi « protégé » pour prévenir l'emploi informel}

La politique économique et sociale menée au lendemain de l'indépendance de l'Algérie (1962) faisait de l'emploi protégé une priorité ; l'emploi informel n'occupait qu'une place marginale. L'indépendance et le socialisme ont été favorables au recul du chômage et à la création d'emplois par l'État. L'emploi informel n'apparaîtra comme un phénomène préoccupant qu'à la faveur de la réforme économique libérale des années 1980 qui n'a pas été sans effet sur l'emploi salarié et l'expansion de l'économie informelle. À partir de 1986, on assiste à une parfaite corrélation entre le taux de chômage croissant, qui atteindra $30 \%$ de la population active en 2001, et celui de l'emploi informel qui se développera à grande échelle. La situation s'améliorera au cours des années 2000, les mesures prises par l'État faisant baisser le taux de chômage progressivement à $10 \%$. Cependant, le poids de l'informel n'a pas cessé d'augmenter à ce jour, atteignant $45 \%$ du total des emplois ${ }^{02}$.

Tel qu'il est observé aujourd'hui, l'encadrement juridique susceptible de contribuer au recul de l'emploi informel porte à la fois sur la formalisation de l'activité de placement, afin de soumettre la demande et l'offre d'emploi au contrôle de l'État (A), et sur l'adoption de mesures pour encourager la création et la déclaration des emplois (B)

\section{A - Formalisation de l'activité de placement}

On ne saurait considérer que l'État est réellement engagé dans une politique pour faire reculer l'emploi informel si, au préalable, il n'intervient pas pour instituer, organiser et contrôler le marché du travail. Entre la fin des années 1980 (début de la transition libérale) et 2004, c'est le marché du travail lui-même qui a connu une part d'activité informelle préjudiciable aux candidats à l'emploi. Cette activité a été le fait de personnes qui s'étaient érigées en opérateurs privés informels dans l'intermédiation entre l'offre et la demande d'emploi. Dans certains cas, sous couvert de courtage, il s'agissait en réalité d'activités illicites correspondant à la définition du marchandage, sans que les pouvoirs publics, pourtant alertés par l'inspection du travail, ne s'en préoccupent. Les travailleurs qui étaient recrutés dans ces conditions d'intermédiation n'étaient pas déclarés à l'organisme de sécurité sociale et ne bénéficiaient pas des garanties du droit du travail. C'est au début des années 2000 que l'État est intervenu pour accompagner la transition libérale, à travers une remise à jour du cadre juridique datant de 1963 et, depuis, tombé en désuétude.

Le législateur a adopté en décembre 2004 une loi relative au placement des travailleurs et au contrôle de l'emploi, afin de réglementer le recrutement et de protéger les candidats à l'emploi. La mise en relation entre l'offre et la demande d'emploi est un service public gratuit. Il est assuré principalement par l'Agence nationale de l'emploi (ANEM). Néanmoins, la loi autorise désormais des organismes privés à concourir au service public du placement sous le contrôle de l'Administration de l'emploi et de l'Inspection générale du travail. Il s'agit pour les pouvoirs publics de libéraliser l'activité de placement, tout en mettant fin, par un encadrement juridique approprié, à ce qui était devenu une activité informelle. Il convient de relever, pour le souligner, qu'en l'état actuel du droit, l'organisme privé de placement est autorisé seulement à fournir des services visant à mettre en relation des offres et des demandes d'emploi, sans qu'il puisse devenir lui-même partie aux relations de travail susceptibles d’en découler. La loi précitée ne

02 Y. Bellache, «Le secteur informel en Algérie : approches, acteurs et déterminants », Les cahiers du CREAD, $\mathrm{n}^{\circ} 105 / 106,2013$, p. 159 et $\mathrm{s}$. 
permet pas la création d'agences privées de fourniture de personnel temporaire, qui recrutent, gèrent et rémunèrent des travailleurs qu'elles enverraient en mission auprès d'entreprises clientes.

Ainsi, l'emploi salarié informel pourrait potentiellement être empêché à la source, à la condition que les services de l'emploi et ceux de l'inspection du travail soient à même d'exercer pleinement leurs prérogatives pour faire respecter les règles les plus récentes qui organisent le fonctionnement du marché du travail.

\section{B - Encouragement à la création d'emplois et à leur déclaration}

Certes, la création d'emplois dépend, avant tout, de l'adoption de mesures en faveur du développement, de l'investissement, de la création d'entreprises et, indirectement, de la promotion de l'emploi salarié ou de l'auto-emploi ${ }^{3}$. Si nécessaire, l'État peut également intervenir par des dispositifs spécifiques lorsque les déséquilibres entre l'offre et la demande persistent. Ces dispositifs se rattachent à la politique sociale. Il s'agit d'assurer, à travers un emploi et sous contrôle de l'Administration des revenus et une couverture sociale à des personnes qui rencontrent des difficultés à accéder au travail salarié ou non salarié dans le cadre du droit commun. Le succès de ces dispositifs peut contribuer à la prévention de l'emploi informel. Depuis la fin des années 1990, les pouvoirs publics ont multiplié et diversifié les actions pour la promotion de l'emploi salarié (1) et le développement du potentiel entrepreneurial (2).

\section{1 - La promotion de l'emploi salarié}

Dans le cadre des mesures visant à encourager la création d’emplois ou, le cas échéant, la déclaration des emplois par les employeurs, il est dérogé au régime des cotisations de sécurité sociale. Une contribution annuelle, prélevée sur le budget de l'État, est destinée à la Caisse de sécurité sociale en contrepartie d'abattements sur la part patronale des cotisations sociales. Cette mesure, pour faire baisser le coût de l'emploi, a pour fondement une loi de décembre 2006 relative aux « mesures d'encouragement et d'appui à la promotion de l'emploi ». Elle permet à tout employeur de bénéficier d'un abattement de $20 \%$ sur la part patronale de la cotisation de sécurité sociale, au titre de chaque recrutement pour une durée égale au moins à douze mois ; ce taux est porté à $28 \%$ pour le recrutement des primo-demandeurs d'emploi et bénéficie à l'employeur tant que la relation de travail est maintenue, dans la limite de trois années. En plus de ces abattements, l'employeur bénéficie pendant trois ans d'une subvention mensuelle à l'emploi pour chaque recrutement dès lors que le contrat de travail est conclu pour une durée indéterminée. Outre ces avantages, en cas de doublement des effectifs, les employeurs occupant neuf travailleurs au moins bénéficient durant une année d'un abattement fixé à $8 \%$ de la quotepart de cotisation à la charge de l'employeur, due au titre des effectifs initiaux employés à la date de mise en œuvre de ce dispositif. De façon plus spécifique encore, dans certaines régions et dans certains secteurs cibles, les recrutements ouvrent droit à des abattements selon des conditions plus avantageuses encore. Enfin, des exonérations globales de contribution à la sécurité sociale sont consenties aux employeurs, pour des périodes allant d'un à trois mois, au titre de la formation ou du perfectionnement accordé aux travailleurs salariés. L'efficacité de ces mesures, dix ans après leur adoption, n'a pas fait l'objet d'une évaluation rendue publique, qui permettrait de connaître leur apport réel au recul de l'emploi informel.

03 A. Boualem Chebira, «Place de l'économie informelle en Algérie et ses impacts sur le processus de développement », Université d'Annaba, Algérie, 2013, 10p., article en libre accès sur http://www.erudite.univparis-est.fr/evenements/colloques-et-conferences/atm-2013-communications-full-papers/?elD=dam frontend push \&docID=25214" 
Au-delà de ces mesures générales, l'État a mis en œuvre progressivement, dès le début des années 1990, des dispositifs spécifiques de création d'emplois temporaires destinés à différentes catégories de jeunes. Ils sont orientés vers la lutte contre l'exclusion sociale et, dans certains cas, vers la transition pour un emploi durable. Ces emplois assurent des revenus garantis par le budget de l'État et la protection sociale.

\section{2 - Développement du potentiel entrepreneurial}

Les restructurations du secteur public économique, durant la période de crise des années 1990, ont eu pour effet un recul de l'emploi salarié protégé par le droit social. Elles ont assurément ouvert la voie au travail et aux activités économiques informelles. Cette tendance résulte d'un a priori idéologique libéral, en opposition à celui qui, des années durant, avait conduit à l'hégémonie de l'entreprise publique, au développement du salariat statutairement protégé et à la marginalisation de l'activité privée.

Le passage de la situation de travailleur salarié à celle de travailleur indépendant emprunte différentes voies. Les années 1990 ont été marquées par un discours alarmiste des pouvoirs publics qui a conditionné les salariés à accepter comme inéluctable et irrésistible la menace généralisée sur l'emploi. C'est pourquoi, aux licenciements massifs qui ont accompagné les restructurations, se sont ajoutés des départs prétendument volontaires et en nombre important de salariés. Des moyens incitatifs soutenant ce « choix » et favorisant le basculement vers un statut d'indépendant ont été adoptés dans la réglementation ou bien négociés directement dans les entreprises. L'option pour le travail indépendant, concernant des salariés ayant perdu leur emploi, est en général encouragée par l'aide publique promise. Il s'agit de leur faciliter l'accès aux facteurs et moyens de production nécessaires à la création de leur activité. Les enquêtes emploi de l'Office national des statistiques (ONS) révèlent ainsi que l'emploi indépendant qui s'est développé dans ce contexte est essentiellement informel (pour deux tiers).

C'est dans ce contexte, où la tendance est à l'expansion de l'auto-emploi informel, que l'État intervient par la mise en place de dispositifs juridiques spécifiques venant en aide à ceux qui portent des projets d'activités économiques viables, mais qui ne peuvent pas les faire aboutir dans le cadre du droit commun des affaires. Des organismes spécialisés ont été créés pour promouvoir des micro-entreprises, avec l'objectif d'en faire des entreprises rentables et durables. Le soutien apporté par l'État porte non seulement sur l'octroi des aides financières nécessaires au lancement des projets, mais aussi, dans les cas de certains dispositifs, sur l'accès au crédit et à la formation à la gestion de l'entreprise.

La Caisse nationale d'assurance chômage (créée en 1994) verse une indemnité mensuelle de chômage aux travailleurs licenciés pour compression des effectifs et leur assure une couverture sociale. Elle est surtout mise à contribution pour favoriser la transition du chômage vers l'activité. Elle organise au bénéfice de ses allocataires des formations dans des Centres de recherche d'emplois et des Centres d'aide au travail indépendant. Depuis 2004, elle a également pour mission le soutien et l'accompagnement à la création de micro-entreprises par les chômeurs promoteurs âgés de 30 à 50 ans $^{04}$. Cet organisme a également établi avec le ministère de la formation et de l'enseignement professionnel, une convention portant sur le soutien à la création de PME par les chômeurs. Divers autres programmes sont développés par cette Caisse. Sans ces programmes, la plupart des travailleurs qui perdent leur emploi

04 Depuis sa création, la CNAC a contribué à la création de 130000 petites entreprises (la part des femmes représente $15 \%$ de cette création) qui ont engendré 200000 emplois. 
dans le secteur formel, en raison d'une compression des effectifs, n'auraient d'autre choix que de se tourner vers des activités informelles.

Un deuxième organisme, l'Agence nationale de soutien à l'emploi des jeunes (créée en 1996), est chargé du soutien et de l'accompagnement des jeunes chômeurs âgés de 19 à 35 ans porteurs de projets de création d'entreprises. Le promoteur bénéficie d'une assistance à titre gracieux, d'aides financières (prêt non rémunéré et bonification des intérêts bancaires), d'avantages fiscaux en phase de réalisation et d'exonérations d'impôts en phase d'exploitation ${ }^{05}$.

Un troisième organisme, l'Agence Nationale de Gestion du micro-crédit (créée en 2004) fournit des services financiers et non financiers adaptés aux besoins des populations sans revenu ou à revenu irrégulier, qui relèvent généralement du secteur informel. Elle vise leur intégration économique et sociale à travers la création d'activités de production de biens et de services, par le biais de prêts non rémunérés favorisant l'auto-emploi. C'est notamment le cas pour le travail à domicile féminin ${ }^{06}$. À ce jour, le salariat demeure cependant la forme d'emploi dominante et concerne en moyenne sept travailleurs occupés sur dix.

\section{II - Extension et effectivité du droit social pour réduire l'informalité}

L'exclusion, en droit ou en pratique, de la protection sociale fait partie de la définition de l'emploi informel. C'est pourquoi la politique de formalisation porte à la fois sur l'extension du droit social à des catégories de travailleurs qui n'entrent pas encore dans son champ d'application et sur son application effective à ceux qui en sont déjà de jure les bénéficiaires. Toutefois, les avancées ne sont pas les mêmes selon qu'il s'agisse du droit du travail (A) ou de celui de la sécurité sociale (B).

\section{A - La protection par le droit du travail}

La portée du droit du travail dans l'économie informelle pose deux questions principales : la première est de savoir si le champ d'application du droit lui-même s'étend à toutes les unités économiques (1). La deuxième porte sur la capacité des pouvoirs publics à faire respecter le droit du travail dans tous les lieux de travail où il a vocation à s'appliquer (2).

\section{1 - Réception du droit du travail dans les activités informelles}

En Algérie, il n'existe ni législation du travail différenciée en fonction des activités, de la taille et du statut juridique de l'entreprise (à l'exception de quelques rares dispositions), ni exonération de l'application de tout ou partie des obligations que cette législation impose visant une catégorie particulière de travailleurs. La distinction entre travail dans l'économie formelle et travail dans l'économie informelle n'est pas une approche pertinente dans la détermination du champ d'application de la législation du travail. En effet, son application s'étend à toute relation de travail salariée, sans autres considérations. La seule distinction qui présente de l'intérêt est celle qui existe entre les travailleurs ayant une relation de travail avec un employeur et les travailleurs indépendants; ces derniers ne sont susceptibles d'être couverts que par les dispositions relatives à la sécurité sociale.

05 Ce dispositif a permis, à ce jour, la création de 375.000 micro-entreprises et 800.000 emplois.

06 À ce jour l'ANGEM a créé 1202270 emplois (dont 62, 52\% pour les femmes). 
Le régime légal de la relation de travail ne fait nullement obstacle à l'application du droit du travail dans l'économie informelle. La législation du travail n'impose aucune exigence de forme pour la conclusion d'un contrat de travail. Selon les termes de la loi, la relation de travail " existe en tout état de cause du seul fait de travailler pour le compte d'un employeur». Aussi, il importe peu que le travail s'effectue dans une unité économique informelle; c'est l'existence d'une relation de travail, constatée ou non par un contrat de travail, qui conditionne l'application du droit du travail et celui de la sécurité sociale au profit du salarié. L'administration de la preuve de l'existence d'une telle relation est facilitée, puisqu'elle peut être apportée par tout moyen par le travailleur qui entend faire valoir ses droits.

Depuis la réforme libérale de 1990, la tendance est à l'extension de la législation du travail à des situations d'activités particulières parmi celles qui, en pratique, favorisent le travail informel. Il en est ainsi de la situation où le travailleur ne fournit pas sa prestation dans une collectivité et semble autonome : travail à domicile pour le compte d'une unité économique. En 1997, la réglementation a doté ces travailleurs d'un statut les assimilant aux travailleurs salariés. Cette qualité permet de leur étendre le bénéfice de toutes les dispositions protectrices applicables aux salariés de droit commun. Il en est ainsi, également, de la situation où le bénéficiaire de la prestation de travail n'est pas « employeur » au sein d'une unité économique : personnels de maison. Cette forme de travail constitue une part du travail informel. Depuis 1990, les personnels de maison sont concernés par l'application du droit du travail. La loi relative aux relations de travail renvoie à un texte réglementaire pour préciser les conditions spécifiques d'emploi de ces personnels; mais ce texte n'a toujours pas été adopté à ce jour. L'emploi, dans ces conditions, reste largement informel, même si le fondement légal pour l'application du droit du travail existe depuis 27 ans. Le champ d'application du droit du travail n'a pas encore été étendu à une autre situation particulière où le travail informel occupe une place appréciable : le travail des aides familiaux. Au moins pour le travailleur à domicile, il est précisé de source réglementaire qu'il peut se faire aider dans ses activités par des membres de sa famille, sans qu'il acquière la qualité d'employeur. De façon générale, le travail des enfants de moins de seize ans est interdit et très peu d'enfants travailleurs semblent être salariés ${ }^{07}$. Néanmoins, le travail des enfants est fréquent et se pratique pour l'essentiel dans de petites entreprises familiales telles que les exploitations agricoles de subsistance, les activités artisanales ou commerciales. L'interdiction de faire travailler des enfants avant l'âge légal (16 ans) devrait s'appliquer même pour des activités développées dans un cadre familial. Le législateur cherche à faire respecter cette interdiction au moyen de l'aggravation de la sanction pénale encourue.

De nouvelles dispositions annoncées par un avant-projet de Code du travail pourraient contribuer à rendre l'accueil de travailleurs informels, dans le cadre de contrats de soustraitance, plus difficile. L'entreprise concernée ne pourrait plus se considérer comme dégagée de toute responsabilité. Il est connu que les PME contournent plus facilement l'application du droit du travail, au détriment des personnes qu'elles font travailler dans ces conditions particulières d'emploi. L'avant-projet de Code du travail prévoit que l'entrepreneur principal et le sous-traitant seront solidairement responsables du respect des prescriptions légales relatives notamment aux conditions de travail, au travail des femmes et des enfants, au travail de nuit, à la sécurité et santé au travail, et à la sécurité sociale.

07 Selon des déclarations du ministère chargé du travail, le travail des enfants existerait à un taux de $0.5 \%$ du nombre total des travailleurs sur les lieux de travail contrôlés. La courbe de l'emploi des enfants en milieu professionnel serait en constante baisse, grâce à l'action de l'inspection du travail, en coordination avec les secteurs composant la commission permanente de protection et de promotion de l'enfant. 
La formalisation du travail peut intervenir aussi en dehors du droit du travail. C'est le cas de l'assistante maternelle à domicile. À l'initiative du ministère de la solidarité (et non du travail), une réglementation est intervenue en 2008 pour formaliser cette relation d'emploi avec les particuliers qui l'emploient. Un contrat doit être établi, sans que le texte réglementaire ne fasse explicitement référence à la loi relative aux relations de travail et ni ne se prononce sur le droit applicable au contrat conclu dans ces conditions ${ }^{08}$. Par contre, la législation sur les assurances sociales leur est applicable. L'observation sociale ne permet pas, cependant, de relever que l'intervention de la réglementation ait fait progresser significativement la formalisation de cette activité très répandue.

\section{2 - Absence du droit du travail dans l'économie informelle}

Il est couramment relevé que la réglementation sur les conditions d'emploi et de travail n'est pas appliquée dans l'économie informelle. Il en est de même de celle qui permet la représentation des travailleurs et la négociation collective. Si cette observation vaut également pour l'Algérie, il faut malheureusement préciser que le non-respect de la législation du travail, en particulier les dispositions relatives aux rapports collectifs de travail, n'est pas le propre des travailleurs informels : c'est une réalité largement partagée avec les travailleurs de l'économie formelle, notamment dans les unités économiques du secteur privé ${ }^{09}$.

Les travailleurs salariés de l'économie informelle, comme d'ailleurs ceux qui travaillent dans des unités de l'économie formelle sans être déclarés, sont généralement réticents à réclamer les garanties du droit du travail, par crainte de perdre leur emploi. Néanmoins, en cas de perte d'emploi, ils sont alors à même d'engager les procédures qui leur permettront d'obtenir les indemnisations et réparations qui leur sont dues; la pratique judiciaire montre qu'ils ne manquent pas de le faire. Mais, lorsqu'il s'agit d'activités informelles fondées sur des relations de parenté ou des relations personnelles ou sociales (par opposition aux relations impersonnelles), le refus de voir ce type de relations inscrit dans le champ du droit du travail viendrait des travailleurs euxmêmes.

En toute circonstance, des efforts doivent être fournis pour faire respecter l'encadrement juridique des conditions d'emploi et de travail. C'est pourquoi, depuis quelques années, ce sont des «brigades mixtes », constituées d'inspecteurs du travail et de contrôleurs de la sécurité sociale, qui sont en charge du contrôle dans les unités économiques informelles ou formelles. En effet, cette distinction n'a aucun impact sur l'exercice de la mission de ces deux corps de fonctionnaires. Leur champ d'action s'étend à tous les lieux de travail, et ceux réputés «informels » n'échappent pas à l'accomplissement de leur mission. Il arrive même qu'ils soient instruits, par leur hiérarchie, pour mener des opérations de jour et de nuit spécialement dans les lieux susceptibles d'employer des travailleurs informels. L'intervention de ces agents s'appuie sur la connaissance qu'ils ont acquise personnellement de l'existence et de l'emplacement de lieux de travail (parfois clandestins) sur le territoire où ils exercent. À l'avenir, selon des dispositions prévues par l'avant-projet de Code du travail précédemment évoqué, il sera fait obligation à tout employeur de déclarer à l'inspection du travail territorialement compétente toute ouverture, fermeture ou transfert d'entreprise, de chantier ou de tout lieu de travail. Cette mesure est destinée à réduire l'informalisation de l'emploi. Toutefois, il est permis de douter que ceux qui ne se soumettent pas, à l'heure actuelle, aux enregistrements et déclarations requis en vertu des législations qui encadrent les activités économiques, se conformeront dans l'avenir à la déclaration que viendrait imposer le Code du travail. Comme pour beaucoup d'autres pays, la question est surtout celle de savoir si les agents

08 K. Mekki, «Régime juridique du travail des assistantes maternelles à domicile », Revue des études juridiques et politiques $n^{\circ} 5$, janvier 2017, Université de Laghouat, Algérie.

09 Le secteur privé absorbe 61,4\% de l'emploi total. 
d'inspection et de contrôle disposent des ressources suffisantes pour accomplir leur mission régulièrement, de manière adéquate, dans toutes les d'entreprises de l'économie formelle ou informelle ${ }^{10}$.

\section{B- La protection par le droit de la sécurité sociale}

L'exclusion de la sécurité sociale est un élément de la définition de l'emploi informel. En Algérie, les dispositions applicables sont issues d'une réforme de 1983. Elles couvrent principalement les travailleurs ayant une relation de travail salariée et accessoirement les personnes assimilées aux salariés (1). Néanmoins, les catégories sociales auxquelles la protection est garantie se sont progressivement diversifiées. L'évolution a permis d'accueillir dans le champ de la sécurité sociale les personnes exerçant à titre indépendant (travailleurs pour leur propre compte et employeurs) et la réglementation la plus récente (2015) permet d'étendre la couverture sociale à des travailleurs à leur propre compte occupés dans des activités reconnues informelles et précaires (2).

\section{1 - Travailleurs salariés et assimilés}

Au regard de la législation relative à la sécurité sociale, le travailleur formel correspond au salarié assuré et le travailleur informel est celui qui n'est pas déclaré à la Caisse nationale des assurances sociales des salariés (CNAS). Il importe peu que l'unité économique soit ellemême considérée comme formelle ou non. C'est l'existence en droit ou en fait d'une relation de travail qui assujettit l'employeur (personne physique ou morale) à la sécurité sociale, avec l'obligation de déclarer son activité et les personnes qu'il emploie. Les travailleurs salariés dans leur ensemble, quels que soient le secteur d'activité (y compris ceux du secteur agricole), le statut juridique de l'entreprise (public ou privé), le régime juridique et la durée de la relation de travail, sont couverts dans les mêmes conditions par la sécurité sociale.

Favoriser la formalité signifie étendre la sécurité sociale, conçue à l'origine principalement pour les agents publics et les salariés, à des catégories de travailleurs qui, en raison de circonstances particulières, n'entraient pas dans le champ d'application du droit du travail, ni consécutivement dans celui des assurances sociales. Une grande réforme des années 1980 a permis cette extension, en fixant par voie réglementaire une liste de huit catégories de personnes assimilées à des salariés en matière de sécurité sociale. Parmi ces catégories, figurent depuis 1985 les travailleurs à domicile et les personnels de maison, avant même que ne leur ait été reconnue la protection du droit du travail (en 1990). Certaines prestations de la sécurité sociale (prestations en nature des assurances maladies et maternité ; prestations en matière d'accidents du travail et de maladies professionnelles) bénéficient également aux porteurs de bagages occupés dans les gares et aux gardiens de parking (non salariés) autorisés à exercer ces activités.

On retiendra de cette évolution qu'il n'y a pas nécessairement superposition entre les champs d'application respectifs du droit du travail et du droit de la sécurité sociale. L'extension de la sécurité sociale a pu, dans certains cas, précéder celle du droit du travail et même, dans d'autres cas, concerner des personnes dont l'activité n'est pas inscrite dans une relation de travail.

10 L'Inspection générale du travail indique avoir effectué en 2016 près de 254.000 visites de contrôle, concernant trois millions de travailleurs. Ces contrôles ont donné lieu à des avertissements et à 47.174 procès-verbaux d'infractions (23\% des entreprises inspectées). 
Ainsi, en droit, il y a eu indéniablement des avancées significatives pour l'extension du champ d'application des assurances sociales ; mais, bien évidemment, cela ne garantit pas que cette extension soit effective, en pratique. En effet, il arrive que l'employeur ne se soumette pas à l'obligation de déclarer son activité. Parfois, alors même qu'il l'a déclarée, il se peut qu'il ne procède pas à l'affiliation de tout ou partie des travailleurs. Il revient alors à ces derniers de mobiliser le droit pour obtenir la régularisation (formalisation) de leur situation. C'est pour encourager davantage les travailleurs concernés à s'engager dans cette voie que l'avant-projet de Code du travail prévoit qu'" outre les obligations prescrites par la législation de sécurité sociale, l'employeur est tenu de notifier au travailleur par écrit son numéro d'affiliation à la sécurité sociale».

Il reste aussi, et surtout, à renforcer la capacité des organismes concernés à faire respecter la loi. Les contrôleurs de la Caisse de sécurité sociale sont quotidiennement sur le terrain pour débusquer les activités et les salariés non déclarés. Ils ont reçu le renfort des inspecteurs du travail ${ }^{11}$. La loi de finances pour 2003 autorise l'administration fiscale à communiquer aux agents des organismes de sécurité sociale et aux inspecteurs du travail tous les renseignements et documents nécessaires à l'accomplissement de leur mission de lutte contre le travail dissimulé. En 2015, les sanctions pénales encourues par l'employeur qui n'a pas procédé à l'affiliation à la sécurité sociale des travailleurs qu'il emploie ont été lourdement aggravées lune amende qui peut atteindre 200000 DA, soit un montant équivalant à 11 fois le salaire national minimum garanti, et/ou une peine d'emprisonnement qui peut atteindre six mois). Les dispositions coercitives peuvent être accompagnées de mesures incitatives. Celles-ci consistent en l'ouverture de périodes de régularisation volontaire au profit des employeurs redevables de cotisations, avec la promesse d'une exonération des majorations et pénalités de retard.

\section{2 - Extension de la sécurité sociale aux travailleurs indépendants}

En Algérie, l'initiative a été prise, au début des années 1990, d'étendre la couverture sociale conçue initialement pour les travailleurs salariés aux indépendants. Leur affiliation à la Caisse nationale de sécurité sociale des non-salariés (CASNOS), créée en 1993, est devenue obligatoire. Plus récemment encore, des mesures ont été adoptées pour adapter les cotisations et les prestations aux caractéristiques des travailleurs engagés dans des activités pour leur propre compte, informelles et précaires.

Dans le cadre du régime général de sécurité sociale, les non-salariés qui exercent une activité industrielle, commerciale, libérale, artisanale, agricole ou toute autre activité, bénéficient dans les mêmes conditions que les salariés des prestations en nature de la sécurité sociale, et selon des dispositions particulières, des prestations en espèces de l'assurance invalidité, de l'assurance décès et de la pension de retraite. Le financement des prestations est assuré par une cotisation à la charge intégrale des bénéficiaires. Le taux de cotisation est fixé à $15 \%$. L'assiette servant de base au calcul est constituée par le revenu annuel imposable dans la limite d'un plafond. Mais, si les employeurs de travailleurs salariés sont réticents à les déclarer, les travailleurs indépendants, eux-mêmes, sont généralement peu enclins à se soumettre à l'obligation de déclaration et à payer les cotisations et cela même lorsque, par ailleurs, ils se sont déclarés au titre de la réglementation régissant leur profession et exercent leur activité dans un cadre formel. C'est pourquoi la prise

11 À titre d'exemple, durant l'été 2017, les inspecteurs du travail et contrôleurs de la sécurité sociale ont inspecté, dans une grande ville, 230 lieux de travail, employant un effectif total de 1150 travailleurs dont 400 travaillent de nuit, notamment dans le secteur des prestations de services, les commerces de vêtements, les restaurants, les hôtels, les pharmacies et les opérateurs téléphoniques. Ils ont dressé 600 mises en demeure et 113 procès-verbaux d'infraction. Ils ont révélé le subterfuge consistant, pour l'employeur, à organiser le travail en deux équipes - jour et nuit - mais à ne déclarer que ceux de l'équipe de jour. 
en compte du critère de la déclaration à l'organisme de sécurité sociale autorise à dire que l'informalité touche plus les non-salariés que les salariés ${ }^{12}$. Comme pour les employeurs, les personnes exerçant une activité non-salariée bénéficient parfois de périodes de grâce pour s'acquitter des cotisations dont ils sont redevables, avec exonération des majorations et pénalités. En vertu d'une convention signée entre la CASNOS et la direction générale des impôts, les personnes qui payent plus pour le compte de la sécurité sociale et qui déclarent moins aux impôts, ne seront pas soumis à un redressement par les services des impôts. Sans donner de précision, pour l'instant, la CASNOS annonce un projet qui regroupera plusieurs départements ministériels, pour inciter les commerçants et les agriculteurs du secteur informel à s'affilier à cette Caisse.

Une initiative plus récente étend le bénéfice de certaines prestations des assurances sociales aux personnes exerçant des activités informelles parmi les plus précaires (comme plusieurs milliers de commerçants ambulants). En effet, une nouvelle mesure, incluse dans la loi de finances complémentaire adoptée en 2015, permet à toute personne occupée, non assujettie à la sécurité sociale, de s'affilier volontairement et de bénéficier, ainsi que ses ayants droit, des prestations en nature de l'assurance maladie et maternité, moyennant le versement d'une cotisation à sa charge. Cette mesure, qui n'est cependant applicable que pour une période transitoire fixée à trois années, a l'effet de permettre au bénéficiaire, selon les termes de la loi, de formaliser son activité.

\section{Conclusion}

La conception du droit social et ses modalités d'application ne sont en aucun cas déterminées par la distinction entre secteur formel et informel. À cet égard, il n'y a aucun sens à prétendre que le droit social ait été conçu pour les salariés du secteur formel et que la formalisation consisterait uniquement dans son extension aux salariés du secteur informel. Il est raisonnable de considérer d'emblée qu'un entrepreneur qui n'inscrit pas son activité dans la formalité au regard des textes qui régissent l'industrie, le commerce ou toute autre activité économique, se montrera également peu soucieux du respect des droits sociaux.

C'est pourquoi favoriser la formalisation signifie surtout accroître les capacités à faire appliquer les lois existantes sur tous les lieux de travail, sans autres considérations. Il n'est pas sûr que la prise en compte de la distinction formel / informel détermine une approche réellement nouvelle du problème habituellement discuté relatif aux obstacles à l'application du droit social et aux conditions à réunir pour en assurer l'effectivité. Ceci met en cause, au premier plan, l'efficacité des institutions étatiques habilitées, mais aussi le rôle des organisations syndicales. Celles-ci ont, en effet, un rôle essentiel à jouer dans cette période de transition libérale en Algérie, pour organiser les travailleurs, afin qu'ils puissent au moins faire valoir les droits qui leur sont légalement reconnus. On ne peut que constater, pour le déplorer, que les travailleurs ne sont organisés en syndicats que dans les entreprises publiques et la Fonction publique, et non dans le secteur privé qui demande pourtant bien plus d'efforts pour y faire respecter la loi. Il reste aussi à réfléchir, sur le plan des rapports individuels de travail, à des prescriptions légales qui seraient de nature à protéger réellement contre la perte d'emploi le salarié qui prend l'initiative d'affronter l'employeur pour faire respecter la loi, en particulier en allant se déclarer lui-même à l'organisme de sécurité sociale.

12 Z. Gasmi, « Secteur informel et protection sociale des non-salariés », Revue Algérienne du Travail, n³1, 2003, p. 69. 


\section{La FORMALISATION DE L'EMPLOI EN ALgéRIE}

Sur la voie de la formalisation, l'extension du champ d'application du droit social à des situations particulières de travail, pour lesquelles il n'a pas été conçu à l'origine, est une de ces questions qui méritent réflexion. Il s'agit principalement de situations où il y a une part d'indétermination lorsqu'il faut faire la distinction entre travail subordonné relevant du contrat de travail et le travail apparaissant comme autonome. Il s'agit aussi de situations où le travail indépendant n'est pas couvert par le droit des assurances sociales, conçu à l'origine pour les salariés. Dans chacune de ces situations, où l'activité se trouve de jure hors du champ d'application des législations garantissant les droits au travail et à la sécurité sociale, il est indéniable que des progrès significatifs sont enregistrés dans la voie de la formalisation. Le droit du travail a étendu son champ d'application à de nouvelles catégories de travailleurs en soumettant leur relation de travail à des régimes spécifiques. Le droit de la sécurité sociale a étendu son champ d'application au-delà du salariat pour couvrir, selon des dispositions particulières, les travailleurs indépendants.

\section{MAHAMMED NASR-EDDINE KORICHE}

Professeur à l'Université d'Alger 3.

Thèmes de recherche : Droits fondamentaux du travail, contrats d'emploi, droit syndical.

Parmi ses publications:

Les transformations du droit algérien du travail / entre statut et contrat, Tome I, Relation d'emploi et de travail : une contractualisation relative, Éd. Office des publications universitaires, Alger, 2009 ;

Les transformations du droit algérien du travail / entre statut et contrat, Tome II, Détermination des conditions de travail : une déréglementation sélective, Éd. Office des publications universitaires, Alger, 2009. 